\section{Pérdida rápida de peso: el caso de los deportes de combate}

\section{Rapid Weight Loss: the case of combat sports}

\section{Sr. Editor:}

Las especialidades deportivas de combate olímpicas, distribuyen a sus atletas en divisiones de peso corporal para favorecer una competencia justa. Sin embargo, muchos atletas utilizan estrategias para perder peso rápidamente, con la intención de competir en divisiones más ligeras ${ }^{1}$.

La pérdida rápida de peso (PRP), corresponde a la reducción del 2 a $10 \%$ del peso corporal la semana previa al pesaje oficial de la competencia, hecho que se acentúa los últimos 2 o 3 días antes del pesaje ${ }^{1}$. Los métodos más comunes para la PRP utilizados por los atletas de las especialidades deportivas de combate son la deshidratación y la restricción de alimentos ${ }^{2}$. En este sentido, el Colegio Americano de Medicina del Deporte ha publicado los potenciales riesgos de estas prácticas ${ }^{3}$. A pesar de ello, la mayoría de estos atletas comienzan con la PRP desde la etapa juvenil2 ${ }^{2}$.

Si bien existen estudios que han demostrado efectos negativos de la PRP sobre el rendimiento físico, no todas las investigaciones han podido corroborarlo ${ }^{4}$. Por otra parte, luego del pesaje oficial, los atletas cuentan con tiempo suficiente para recuperar parte de su peso corporal antes de la competencia ${ }^{5}$, lo que disminuiría la potencial merma de su rendimiento físico. Por ejemplo, en judo se ha observado que los atletas medallistas recuperan mayor peso luego del pesaje oficial en comparación a los atletas no medallistas ${ }^{5}$, sin embargo, esta situación no se ha reportado en boxeadores ${ }^{6}$. Adicionalmente, Artioli et al. han planteado que la PRP tiene implicancias éticas ${ }^{1}$, debido a la influencia que podría generar un atleta que combate en divisiones más bajas respecto de su peso de entrenamiento, sobre el resto de atletas (efecto cascada), quienes replicarían la misma conducta con el afán de equiparar las condiciones de competencia. Los mismos autores señalan que la PRP podría ser considerada doping, ya que cumple con tres de los criterios contemplados por la Agencia Mundial Antidopaje para las sustancias prohibidas ${ }^{1}$, estas son: 1) mejora el rendimiento, 2) pone en peligro la salud del atleta, y 3) viola el espíritu del deporte.

En consideración a los antecedentes antes expuestos, algunas federaciones deportivas de combate olímpicas, como el judo y la lucha, han realizado modificaciones a sus reglamentos. En el caso del judo, el pesaje se realiza el día anterior a la competencia, pero además, el mismo día de la competencia, se incluyó un nuevo pesaje aleatorio donde el atleta no puede exceder más de $5 \%$ de su división de competencia, de lo contrario, es descalificado. Por otro lado, la lucha olímpica, trasladó el pesaje oficial para el mismo día de la competencia, para evitar las fluctuaciones de peso que antecedían a esta normativa.

En Chile, no contamos con información sobre la prevalencia y variedad de estrategias que utilizan los atletas de las especialidades deportivas de combate olímpicas para la pérdida de peso. Datos de este tipo, serían un gran aporte para educar a los atletas desde su etapa de formación, así como para los entrenadores, técnicos y equipos médicos que podrían contar con dietas acordes a las necesidades de la especialidad, biotipo y características individuales de los atletas. Toda vez que, esta es una realidad que atenta contra la salud de las personas ${ }^{3}$. En este sentido, el propósito de esta carta es alertar sobre los riesgos de la PRP y respecto a la necesidad de especialistas en Nutrición deportiva que, puedan emprender las acciones de prevención, evaluación, seguimiento y educación de atletas, entrenadores, técnicos y familias con la intención de favorecer la salud y el rendimiento deportivo. Sin embargo, hasta donde sabemos, existe sólo un Magister en nutrición para la actividad física y el deporte, impartido por la Universidad Mayor y, dos diplomados en nutrición deportiva, impartidos por la Universidad Finis Terrae y la Universidad San Sebastián respectivamente. Mientras que otros profesionales de la salud como los médicos, ya cuentan con la Especialidad de Medicina del Deporte, impartida por la Universidad Mayor. Por lo tanto, contar con profesionales de la Nutrición formados para desenvolverse en el contexto deportivo, particularmente, con deportes de combate, es urgente. Debido a la abundante práctica de este tipo de disciplinas en el país, y por las proyecciones deportivas que podría lograr Chile cuando organice los Juegos Panamericanos de 2023, dado a que 20\% de las medallas de oro se reparten en estos deportes.

Esta situación se agrava al considerar que en los Megaeventos (i.e. Juegos Suramericanos, Panamericanos y Olímpicos) algunos deportes de combate reducen el número de divisiones de peso. Por ejemplo, el taekwondo cuenta con 16 categorías (8 para hombres y 8 para mujeres) para las competencias internacionales de la especialidad, no obstante, para los Megaeventos las divisiones disminuyen a la mitad (4 para hombres y 4 para mujeres), lo que aumenta el riesgo de requerir la PRP por parte de los atletas.

Para enfrentar en parte este problema, proponemos:

Incorporar una unidad sobre nutrición en deportes de combate en los programas de estudio de las carreras de Nutrición y Dietética, así como en los Postgrados de Nutrición Deportiva.

Los nutricionistas podrían emplear como instrumento diagnóstico para la PRP, el Rapid Weight Loss Questionnaire $R W L Q$, ya que otorga un índice sobre la gravedad de los métodos de PRP y cuenta con un extendido uso en la literatura científica ${ }^{2}$. 
Los nutricionistas deberían considerar además del peso corporal y variables antropométricas, el estado de hidratación de los atletas valorado a través de pruebas de densidad de orina.

Incorporar nutricionistas en los programas de formación deportiva para educar desde temprana edad a los atletas, familias y equipos técnicos sobre alimentación saludable, y preparar a los atletas individualmente respecto a cómo enfrentar el pesaje sin riesgo.

Finalmente, reforzamos el llamado a la especialización de los nutricionistas sobre un tema tan sensible para el deporte como es la PRP, pero a la vez, extendemos la invitación al resto de profesionales involucrados en este ámbito como médicos, kinesiólogos, licenciados en Ciencias de la Actividad Física y profesores de Educación Física para trabajar en conjunto, no solo en favor del desarrollo del deporte, sino que también, en el ámbito de la salud, promocionando la práctica regular de actividad física.

Tomás Herrera-Valenzuela ${ }^{1,2,3, a, d, e}$, Pablo Valdés-Badilla ${ }^{4,5,6, a, d}$, Estefanía Soto-Voisier ${ }^{7,8, b}$, Francisco Verdugo-Miranda ${ }^{8,9,}$, Jorge Cancino-López ${ }^{10, a, e}$, Mauricio Sáez-Fuentes ${ }^{8, a, d}$, Cristian Cofre-Bolados ${ }^{2,3, a, d, e}$, Samuel Durán-Agüero ${ }^{11, b, d, e}$, Pedro A Orihuela ${ }^{1, c, e}$ ${ }^{1}$ Laboratorio de Inmunología de la Reproducción, Facultad de Química y Biología, Universidad de Santiago de Chile (USACH), Santiago, Chile.

${ }^{2}$ Laboratorio de Ciencias de la Actividad Física, el Deporte y la Salud, Facultad de Ciencias Médicas, Universidad de Santiago de Chile (USACH), Santiago, Chile.

${ }^{3}$ Escuela de Ciencias del Deporte, Facultad de Salud, Universidad Santo Tomás (UST), Santiago, Chile. ${ }^{4}$ Instituto de Actividad Física y Salud, Universidad Autónoma de Chile, Temuco, Chile. ${ }^{5}$ Pedagogía en Educación Física, Facultad de Educación, Universidad Autónoma de Chile, Temuco, Chile. ${ }^{6}$ Programa de Doctorado en Ciencias de la Actividad Física, Facultad de Ciencias de la Educación, Universidad Católica del Maule, Talca, Chile. ${ }^{7}$ Carrera de Nutrición y Dietética, Facultad de Medicina, Universidad del Desarrollo, Santiago, Chile. ${ }^{8}$ Unidad de Ciencias del Deporte, Centro de Alto Rendimiento, Santiago, Chile.
${ }^{9}$ Centro de Salud Deportiva, Clínica Santa María, Santiago, Chile.

${ }^{10}$ Laboratorio de Ciencias del Ejercicio, Escuela de Kinesiología, Facultad de Medicina, Universidad Finis Terrae, Santiago, Chile.

${ }^{11}$ Facultad de Ciencias de la Salud. Universidad San Sebastián. Chile. aProfesor de Educación Física.

${ }^{b}$ Nutricionista. 'Licenciado en Ciencias Biológicas. ${ }^{d} \mathrm{MsC}$. ${ }^{e} P h D$ fPediatra.

\section{Referencias}

1. Artioli GG, Saunders B, Iglesias RT, Franchini E. It is Time to Ban Rapid Weight Loss from Combat Sports. Sport Med 2016; 46 (11): 1579-84.

2. Artioli GG, Gualano B, Franchini E, Scagliusi FB, Takesian M, Fuchs, et al. Prevalence, Magnitude, and Methods of Rapid Weight Loss among Judo Competitors. Med Sci Sport Exerc 2010; 42 (3): 436-42.

3. Oppliger RA, Case HS, Horswill CA, Landry GL, Shelter AC. American College of Sports Medicine position stand. Weight loss in wrestlers. Med Sci Sports Exerc 1996; 28 (6): ix-xii.

4. Yang W, Heine O, Mester J, Grau M. Impact of rapid weight reduction on health and performance related indicators of athletes representing the Olympic combat sports. Arch Budo 2017; 13: 147-60.

5. Reale R, Cox GR, Slater G, Burke LM. Regain in Body Mass After Weigh-In is Linked to Success in Real Life Judo Competition. Int J Sport Nutr Exerc Metab 2016; 26 (6): 525-30.

6. Zubac D, Karnincic H, Sekulic D. Rapid Weight Loss is Not Associated With Competitive Success in Elite Youth Olympic-style Boxers in Europe. Int J Sports Physiol Perform 2017; $28: 1-27$.

Correspondencia a:

Tomás Herrera-Valenzuela

Las Sophoras 175, Estación Central, Santiago, Chile. tomas.herrera@usach.cl

Conflicto de interés: No presenta. 